\title{
Exploring the Relationship between Web Accessibility and User Experience
}

DOI:

10.1016/j.ijhcs.2016.03.008

\section{Document Version}

Accepted author manuscript

Link to publication record in Manchester Research Explorer

\section{Citation for published version (APA):}

Aizpurua, A., Harper, S., \& Vigo, M. (2016). Exploring the Relationship between Web Accessibility and User Experience. International Journal of Human-Computer Studies, 91, 13-23.

https://doi.org/10.1016/j.ijhcs.2016.03.008

\section{Published in:}

International Journal of Human-Computer Studies

\section{Citing this paper}

Please note that where the full-text provided on Manchester Research Explorer is the Author Accepted Manuscript or Proof version this may differ from the final Published version. If citing, it is advised that you check and use the publisher's definitive version.

\section{General rights}

Copyright and moral rights for the publications made accessible in the Research Explorer are retained by the authors and/or other copyright owners and it is a condition of accessing publications that users recognise and abide by the legal requirements associated with these rights.

\section{Takedown policy}

If you believe that this document breaches copyright please refer to the University of Manchester's Takedown Procedures [http://man.ac.uk/04Y6Bo] or contact uml.scholarlycommunications@manchester.ac.uk providing relevant details, so we can investigate your claim.

\section{OPEN ACCESS}




\title{
Exploring the Relationship between Web Accessibility and User Experience
}

\author{
Amaia Aizpurua $^{\mathrm{a}, \mathrm{b}, *}$, Simon Harper ${ }^{\mathrm{b}}$, Markel Vigo ${ }^{\mathrm{b}}$ \\ ${ }^{a}$ Faculty of Informatics, University of the Basque Country, UPV/EHU, \\ Donostia-San Sebastián, Spain \\ ${ }^{b}$ School of Computer Science, University of Manchester, \\ Manchester, United Kingdom
}

\begin{abstract}
Understanding the interplay between the user experience (UX) and Web accessibility is key to design Web sites that, beyond access, could provide a better UX for people with disabilities. In this paper we examine the relationship between UX attributes and Web accessibility. We measured accessibility in two ways: the perceived accessibility as reported by participants and accessibility in terms of conformance to guidelines. Findings uncover that perceived Web accessibility is significantly correlated with 27 of the 35 UX attributes analysed, suggesting these two qualities are closely related. The relationship between UX and conformance to WCAG 2.0 is more elusive: we only found significant correlations between the hedonic attributes original, innovative and exciting.
\end{abstract}

Keywords: Blind users, Screen readers, Web, Web accessibility, User

Experience

\section{Introduction}

The World Wide Web has an incredible potential to make our lives better due to the wide range of services offered through it. The Web can be specially helpful for people with disabilities, as barriers to communication and interaction that many people face in the physical world are removed. While the Web was

\footnotetext{
* Corresponding author

Email address: amaia.aizpurua@manchester.ac.uk (Amaia Aizpurua)
}

Preprint submitted to International Journal of Human Computer Studies

April 11, 2016 
designed to be universally accessible, in practice this does not always happen (Lopes et al. 2010) mainly because Web sites are often designed without considering human diversity. This leads to poorly designed Web sites which can potentially exclude significant segments of the population. Since the Web is a mainly visual environment, navigating the Web is particularly challenging for blind users. Although assistive technology such as screen readers have been an incredible breakthrough, blind users still face a wide range of difficulties on the Web. In fact, blind users face not only more challenges than sighted users (Bigham et al., 2007), but are also disadvantaged when compared to other groups of users with disabilities (Petrie et al., 2004).

The World Wide Web Consortium (W3C) through the Web Accessibility Initiative (WAI) published the Web Content Accessibility Guidelines (WCAG) (Chisholm et al., 1999, Caldwell et al., 2008) to promote the design of accessible Web content. While guidelines are an invaluable starting point, prior empirical research (Power et al., 2012) indicates that WCAG 2.0 only cover around half of the problems that blind users encounter on the Web. This implies that a Web site may have an adequate level of accessibility in terms of conformance to guidelines, but still not be sufficient for users. Our experience, which is informed by a series of studies with blind participants, corroborates that a Web site with 25 a significant number of WCAG 2.0 success criteria violations can be perceived to be accessible; on the contrary, a Web site which is compliant to guidelines may not be always perceived to be accessible (Aizpurua et al., 2013).

Research on the behavioural aspects of blind users on the Web has been mainly focused on the analysis of performance in terms of efficiency, errors committed and satisfaction (Leuthold et al., 2008), and on examining the strategies employed to overcome the barriers they encounter and the situations that trigger the use of coping tactics (Vigo \& Harper, 2013). These works have provided a valuable knowledge about how blind users behave and navigate on the Web although they say little about the 'intangible' aspects of the experience. In other words, since behaviour is accompanied by subjective experience, in addition to assessing objective qualities such as performance-related aspects of the interac- 
tion, the interaction with Web sites should be explored in a more holistic way. In this respect user experience (UX) provides a framework to understand how users may perceive an interactive artefact from diverse facets including aesthetics, af40 fect or trust (Law et al., 2009). We claim that having a better understanding of blind users' subjective experience on the Web cannot be disassociated from an analysis of how this experience affects the perceived accessibility.

To shed some light on this association we examined the relationship between the UX and Web accessibility by comparing the subjective experience 45 reported by users, with the perceived Web accessibility and with conformance to accessibility guidelines. User experience scores were collected by means of questionnaires and semi-structured interviews based on the UX model proposed by Hassenzahl (2005). In Section 4.1 we examined the relationship between UX and perceived Web accessibility after-use and found that perceived Web 50 accessibility (PWA) is significantly correlated with most of the UX attributes, suggesting the close relationship between these qualities. Secondly, we investigated how UX attributes are related to different Web Accessibility Indicators (AIs), which were measured using different accessibility evaluation methods. The outcomes of this analysis reveal in Section 4.2 that compliance to WCAG 2.0 guidelines is significantly correlated to three UX attributes that belong to the hedonic quality: original, innovative and exciting. In Section 4.3 we analysed the interviews with participants in order to provide possible explanations for these relationships. Finally, results and implications are discussed in Section 5. This work is not only novel due to the number of covered UX attributes, but also because we study the experience of blind users with regard to a more holistic view of Web accessibility, as the subjective perception of participants and a more normative assessment of accessibility have been considered. 


\section{Background}

\subsection{The Importance of the Experience}

Usability is a clearly defined concept: the ISO 9241-11 (ISO, 1998) defines usability as "the extent to which a product can be used by specified users to achieve specified goals with effectiveness, efficiency and satisfaction in a specified context of use". However, Web sites are much more than interactive artefacts for accomplishing specific tasks. Individuals find the Web as a mean, not only to achieve informational goals, but also for activities related to communication, leisure, social networking or contributing to building the Web (Lindley et al. 2012). Users value interactive artefacts on the basis of how well they satisfy their needs in a particular situation, beyond the objective features that derive from design choices including content, style or functionalities. Therefore, the

75 success of a Web site may not only depend on its actual characteristics, but also on how well these are perceived by users (Hassenzahl, 2005). This highlights the importance of considering the subjective aspects of the interaction in order to understand the actual experience of users. Because the HCI community has acknowledged the importance of these non-instrumental aspects, several works have focused on defining and setting the scope of UX: according to a survey, UX is considered dynamic, context-dependent and subjective and therefore, difficult to agree upon (Law et al., 2009). Similarly, Bargas-Avila \& Hornbæk (2011) found a lack of consistency in the methods employed to evaluate different UX attributes. This is a symptom of not having a common framework that allows researchers and practitioners to understand the UX attributes and the relationships among them. So far, only a few models have been proposed, for instance, the most comprehensive ones are the UX model (Hassenzahl, 2005) and the CUE model (Thüring \& Mahlke, 2007). Both models share the same foundations, as both include instrumental and non-instrumental qualities, the 90 emotional reactions of users and their appraisal of interactive artefacts. 


\subsection{Web Accessibility, Usability and $U X$}

There is little agreement when it comes to defining web accessibility, which causes some tensions between the community of users, researchers and accessibility advocates (Yesilada et al., 2012). Moreover, while it cannot be denied

\subsection{The Experience of Blind Users on the Web}

In order to understand the experience of blind users on the Web, previous ${ }_{\text {}}$ work has focused on identifying the problems they encounter (Theofanos \& Redish, 2003, Murphy et al., 2008, Power et al., 2012), analysing their performance ${ }_{120}$ (Leuthold et al. 2008), examining their navigation behaviour and the coping 
strategies they use (Vigo \& Harper, 2013). Works about the subjective aspects of the interaction of blind users with the Web are scarce: Lazar et al. (2006) conducted a study that examined the frustrating experiences and mood changes of 100 participants with visual disabilities when browsing the Web. One of the main findings showed that frustration causes individuals' mood to deteriorate. More specifically, the factors that had the strongest negative impact on mood were those related to the ability to complete the work.

\section{Method}

\subsection{Participants}

Eleven participants ( 4 females and 7 males) took part in the study, as shown in Table 1. The median age of participants was 43 years, with a range of 21-64 years. Expertise varied among participants: 2 were experts, 2 beginners, 4 intermediates and 3 advanced. In an interview which took place before the navigation tasks we asked participants to rate their expertise on the Web on a four-item scale: expert, advanced, intermediate and beginner. Due to the observed lack of reliability of self-reported values, Web expertise was also assessed based on external observation of the navigation skills shown by participants. The first author looked at the strategies employed and the confidence of participants when carrying out the proposed tasks. These assessments were corroborated by the facilitator in charge of the computer training facility at the National Organisation of Spanish Blind People (ONCE), who had trained the participants. In line with the findings of van der Geest et al. (2014), who found that self-rated competence of visually impaired users is not always related to their actual performance, Table 1 shows that self-rated and observed skills do not necessarily match.

\subsection{Apparatus}

All participants were legally blind and utilised screen readers to navigate on the Web: ten participants were Jaws users (version 10, except for P01 and P03 
Table 1: Demographic data and characterisation of Web expertise

\begin{tabular}{ccccccc}
\hline part. id & gender & age & Web familiarity & frequency of Web use & \multicolumn{2}{c}{$\begin{array}{c}\text { expertise } \\
\text { observed }\end{array}$} \\
\hline P01 & f & 29 & $>7$ years & daily & advanced & expert \\
P02 & f & 29 & $>7$ years & daily & advanced & expert \\
P03 & m & 39 & $>7$ years & daily & advanced & advanced \\
P04 & m & 54 & $4-6$ years & daily & intermediate & intermediate \\
P05 & m & 43 & $1-3$ years & weekly & beginner & intermediate \\
P06 & m & 21 & $1-3$ years & weekly & beginner & beginner \\
P07 & m & 64 & $>7$ years & daily & intermediate & advanced \\
P08 & m & 58 & $>7$ years & daily & intermediate & intermediate \\
P09 & $\mathrm{f}$ & 54 & $4-6$ years & daily & intermediate & advanced \\
P10 & $\mathrm{m}$ & 64 & $4-6$ years & daily & beginner & intermediate \\
P11 & $\mathrm{f}$ & 42 & $>7$ years & weekly & intermediate & beginner \\
\hline
\end{tabular}

who used version 12) on Internet Explorer and Windows XP and Windows 7 respectively, while just one participant (P02) was a VoiceOver user on Safari over MacOS. The first three participants were observed in the research facility of the HCI laboratory at the School of Computer Science of the University of the Basque Country, where they brought their own laptop. Remaining sessions took place in a room at the ONCE delegation in Donostia-San Sebastián, where these eight participants used the same laptop and keyboard, which were provided by the ONCE.

\subsection{Stimuli Selection}

We recruited local participants and selected Web sites of restaurants that were popular in the area where participants lived in order to let the subjective dimensions emerge. We focused on one type of Web site for two main reasons: 1) to establish analogous tasks across different stimuli; and 2) to minimise potential confounding factors resulting from different types of Web sites.

In order to select the final set of Web sites we first listed 25 Web sites of local restaurants of different styles. We then analysed their homepages, using four automated Web accessibility evaluation tools: AChecker (Gay \& Li, 2010), 
EvalAccess (Abascal et al. 2004), TAW 1 and WAVE2 Using the WAQM metric (Vigo et al. 2007) we evaluated again the homepage and two more Web pages. Based on the results we classified the Web sites into 2 groups: highly accessible and poorly accessible sites. Within each group we separated Web sites into two other groups considering the style of the restaurant: traditional and innovative. Then we performed manual accessibility evaluations: we applied the Barrier Walkthrough (BW) inspection method (Brajnik, 2006) in three Web pages of each Web site. Based on the results, we finally selected the two most and least accessible Web sites for each type of restaurant.

We evaluated the final four Web sites against the AA conformance level of WCAG 2..$^{3}$ The selected Web sites contained different features and problems: in general, W1 and W2 satisfied more AA level success criteria (SC) than W3 and $\mathrm{W} 4$. The homepages of $\mathrm{W} 1, \mathrm{~W} 2, \mathrm{~W} 3$ and $\mathrm{W} 4$ satisfied respectively $73 \%$, $69 \%, 52 \%$ and $36 \%$ of the SC for the AA level of WCAG 2.0. The most severe accessibility problem of W3 was that the seven links that conform the navigation menu, all of them have the same text which is 'image'. In the case of W4, the main accessibility problem is about Flash content which is not accessible using a screen reader.

Regarding the above-mentioned selection criteria with respect to branding, W1 and W3 represent internationally well-known restaurants with an innovative character and a culinary style based on research and creativity. In contrast, W2 and W4 Web sites correspond to restaurants that are locally popular and their style is based on traditional Basque cuisine. The visual design of the Web sites is in line with the style of the restaurant. The Web sites of the internationally well-known restaurants (W1 and W3) have more stylised visual layouts, as they have had their typographies designed and make use of high-quality close-up pictures. In contrast, the Web sites of the traditional-style restaurants (W2

\footnotetext{
1 http://www.tawdis.net

2 http://wave.webaim.org/

${ }^{3}$ Both VoiceOver and Jaws screen readers were used on BW and WCAG 2.0 evaluation.
} 


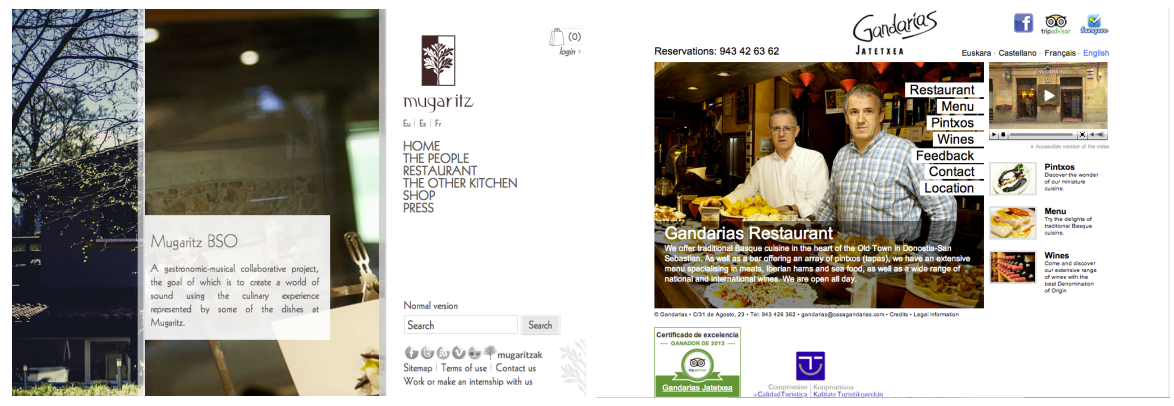

(a) W1: high accessibility and innovative (b) W2: high accessibility and traditional
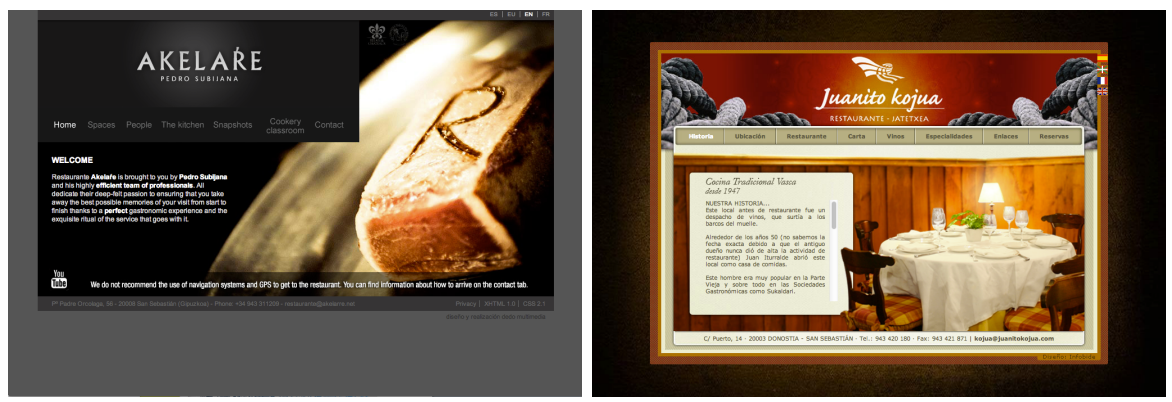

(c) W3: low accessibility and innovative (d) W4: low accessibility and traditional

Figure 1: Screenshots of Web sites.

and W4) have a more basic and less elaborated visual aesthetic designs (see a snapshot of their homepages in Figure 1).

\subsection{Procedure}

Each session was conducted with one participant at a time. Once the participants were informed about the objectives of the study and the procedure of the session they signed a consent form. In order to reduce bias in the obtained answers we told participants that we had no conflict of interests with the Web sites, and that we had only selected those Web sites for the purpose of the study. We let the participants know that we were interested in their personal opinions in order to let them play the role of testers rather than tested subjects. We also made it clear that there were no right or wrong answers. Thus, they would feel free to respond as honestly as possible. 
Then, we asked each participant questions about demographics, and their Web expertise, including their familiarity with the Web and the frequency of access to the Web. After that, each participant was interviewed about his previous experiences and expectations regarding restaurant Web sites. Once the interview had finished, the participant could start to navigate the first Web site. Following a within-subject design, each participant was asked to complete the same three consecutive tasks within each Web site (more in Section 3.5). Repeating tasks on all Web sites did not introduced a potential learning effect bias as each Web site structured its content in a different manner. In order to minimise order effects, Web site navigation order was counterbalanced. Once they finished the tasks or withdrew from them, participants were asked to rate the items of the questionnaires (more in Section 3.6). Then they were interviewed about their browsing experience. Each session, including the navigation on Web sites and interviews, was video and audio recorded to enable subsequent analysis.

\subsection{Tasks}

The three tasks were: 1) freely navigate on the Web site in order to become familiar with it; 2) find information about the gastronomic offer; and 3) find the means offered by the Web site to make a booking. The idea was to let the users explore the Web site through real tasks, which would induce a more naturalistic behaviour. Even if tasks were set in advance, they were not very specific and were kept open, as participants were not given explicit clues or directions to follow in order to find the information. This allowed the participants to explore and browse each Web site with ample opportunities. Participants were told that the time estimated for each task was between 5-10 minutes. However we insisted on their freedom to spend more time or withdraw from navigating if they wanted. For this reason completion times were irrelevant and not useful for the scope of our study. 


\subsection{Data Collection}

We used existing instruments to capture the UX of participants in order

235 had. The instruments we selected after reviewing the literature were: Attracdiff 2 (Hassenzahl, 2008) and the emotion word prompt list (EWPL) by Petrie \& Precious (2010) both in Table 2. Attracdiff 2 was used for collecting the insights from participants about the Web sites. This questionnaire consists of a

intensity. We translated both questionnaires into Spanish.

Information about the Web accessibility perceptions of participants was obtained by asking participants to rate the accessibility of each Web site in a 7-point Likert-type question, from 1 (very inaccessible) to 7 (very accessible).

collected by the questionnaires. Some of the prompts we gave them aimed at knowing more about the moment and the reasons for their emotional reactions, the problems they encountered while navigating, the positive and negative aspects of the Web sites, etc.

The accessibility indicators (AIs) were obtained applying the different evaluation methods described in Section 3.3. As a result, we computed 37 accessibility indicators that derived from four main sources: the TAW online automated 
evaluation too ${ }^{4}(t o o l)$, the metrics from the WAQM software $(m)$ and those obtained through inspection methods: conformance to WCAG $2.0(s c)$ and the Barrier Walkthrough method $(b w)$. These four main AIs were broken down into more specific indicators resulting in a list of 37 AIs: the automatically detected problems $(e)$, warnings that require manual verification $(w)$; the four accessibility principles of the WCAG 2.0 guidelines: perceivable $(p)$, operable $(o)$, understandable $(u)$ and robust $(r)$; the conformance level $(a, a a)$ and the number of satisfied (sat) and not-satisfied (nsat) success criteria $(s c)$. For instance, $m$ represents the average accessibility score computed by WAQM, tool_ $w_{-} p$ refers to the number of warnings reported by the tool for the $p$ principle, tool_e_ $u$ corresponds to the number of errors reported by the tool for the $u$ principle, $s c_{-} s a t \_a$ represents the number of satisfied $s c$ for the A conformance level, $s c_{-} n s a t_{-} a a_{-} p$ 275 indicates the number of not satisfied $s c$ for the AA conformance level for the $p$ principle, and $b w$ corresponds to the number of barriers found using the Barrier Walkthrough method.

\subsection{Data Analysis}

We run analyses of statistical correlation to observe the relationships between: 1) UX attributes and perceived Web accessibility (PWA) as reported by participants; and 2) UX attributes and accessibility indicators (AIs) as generated by tools and inspections by experts. The statistical software used was $\mathrm{R}^{5}$ We also analysed data from the interviews in order to better understand the importance of the identified relationships and the reasons why they emerged.

\footnotetext{
${ }^{4}$ We chose the TAW online tool because it provides a straightforward way to discriminate automatically reported violations and warnings that require human verification.

${ }^{5}$ http://www.r-project.org/
} 


\subsection{UX and Perceived Web Accessibility}

Table 2 shows that most correlations between PWA and UX attributes (27 out of 35) are statistically significant when we compute Kendall's Tau test ${ }^{6}$ We found strong significant $(\tau>0.5$ and $p<0.001)$ correlations between PWA and six attributes of the hedonic quality-identification (integrating, professional, valuable, inclusive, brings me closer to people, presentable), one attribute of the hedonic quality-stimulation (creative), five attributes of the pragmatic quality (simple, practical, direct, clear, manageable), goodness, appeal, annoyed, disappointed, frustrated, happy, interested and pleased. We also found significant and moderate correlations $(0.4 \geqslant \tau \geqslant 0.5$ and $p<0.001)$ between PWA and one attribute of hedonic quality-identification (classy), three attributes of hedonic quality-stimulation (original, exciting, new), beauty, bored and confused.

\subsection{UX and Accessibility Indicators}

We examined the relationship between the UX attributes rated by participants and the accessibility indicators corresponding to Web sites. Since Likert scales can actually be considered somewhere between an ordinal and a true interval scale (Maxwell, 2006), it has been a subject of debate for years how these scales should be appropriately analysed (Carifio \& Perla, 2007; Norman, 2010). Due to the exploratory nature of our research, we decided to apply both correlation tests. If we focus on large effect sizes (above 0.5) the Pearson and Spearman test yielded some significant correlations, while the Kendall test did not. Table 3 shows that, predominantly, conservative-innovative (HQS_4) and lame-exciting (HQS_5) attributes are the ones with more strong and significant correlations. We observe that these correlations correspond to those criteria in which conformance to WCAG 2.0 guidelines was evaluated by human testers,

\footnotetext{
${ }^{6}$ Kendall's Tau is preferred to Spearman test because it performs better with small sample sizes (Clark-Carter 2004).
} 
Table 2: Correlations between PWA and UX attributes for the Kendall test $[\mathrm{N}=44,11$ participants x 4 Web sites]. Significance of tests are reported at $p<0.05\left(^{*}\right), p<0.005\left(^{* *}\right)$, $p<0.001(* * *)$.

\begin{tabular}{|c|c|}
\hline UX attributes & Kendall's Tau $(\tau)$ \\
\hline \multicolumn{2}{|l|}{ Attracdiff - Hedonic quality-identification } \\
\hline HQI_1 isolating-integrating & $0.63^{* * *}$ \\
\hline HQI_2 amateurish-professional & $0.64^{* * *}$ \\
\hline HQI_3 gaudy-classy & $0.44^{* * *}$ \\
\hline HQI_4 cheap-valuable & $0.62^{* * *}$ \\
\hline HQI_5 noninclusive-inclusive & $0.68^{* * *}$ \\
\hline HQI_6 takes me distant from people-brings me closer to people & $0.66^{* * *}$ \\
\hline HQI_7 unpresentable-presentable & $0.67^{* * *}$ \\
\hline \multicolumn{2}{|l|}{ Attracdiff - Hedonic quality-stimulation } \\
\hline HQS_1 typical-original & $0.43^{* * *}$ \\
\hline HQS_2 standard-creative & $0.55^{* * *}$ \\
\hline HQS_3 cautious-corageous & 0.18 \\
\hline HQS_4 conservative-innovative & $0.29^{*}$ \\
\hline HQS_5 lame-exciting & $0.47^{* * *}$ \\
\hline HQS_6 easy-challenging & -0.19 \\
\hline HQS_7 commonplace-new & $0.45^{* * *}$ \\
\hline \multicolumn{2}{|l|}{ Attracdiff - Pragmatic Quality } \\
\hline PQ_1 technical-human & $0.37^{* *}$ \\
\hline PQ_2 complicated-simple & $0.52^{* * *}$ \\
\hline PQ_3 impractical-practical & $0.64^{* * *}$ \\
\hline PQ_4 cumbersome-direct & $0.53^{* * *}$ \\
\hline PQ_5 unpredictable-predictable & $0.39^{* *}$ \\
\hline PQ_6 confusing-clear & $0.55^{* * *}$ \\
\hline PQ_7 unruly-manageable & $0.60^{* * *}$ \\
\hline \multicolumn{2}{|l|}{ Attracdiff - Evaluational Constructs } \\
\hline beauty & $0.48^{* * *}$ \\
\hline goodness & $0.68^{* * *}$ \\
\hline appeal & $0.61^{* * *}$ \\
\hline \multicolumn{2}{|l|}{ EWPL } \\
\hline annoyed & $-0.56^{* * *}$ \\
\hline bored & $-0.46^{* * *}$ \\
\hline confident & 0.10 \\
\hline confused & $-0.47^{* * *}$ \\
\hline disappointed & $-0.58^{* * *}$ \\
\hline frustrated & $-0.58^{* * *}$ \\
\hline happy & $0.59^{* * *}$ \\
\hline interested & $0.54^{* * *}$ \\
\hline hopeful & $0.41^{* *}$ \\
\hline pleased & $0.54^{* * *}$ \\
\hline unsure & $-0.26^{*}$ \\
\hline
\end{tabular}


and specially the ones related to the number of satisfied and non-satisfied success criteria for the Perceivable principle.

Table 3: Correlations between Accessibility Indicators and UX attributes for the Pearson test $[\mathrm{N}=88,11$ participants $\mathrm{x} 4$ Web sites $\mathrm{x} 2$ Web pages $)]$ for $\mathrm{p}<0.001$. Values between brackets correspond to the Kendall test.

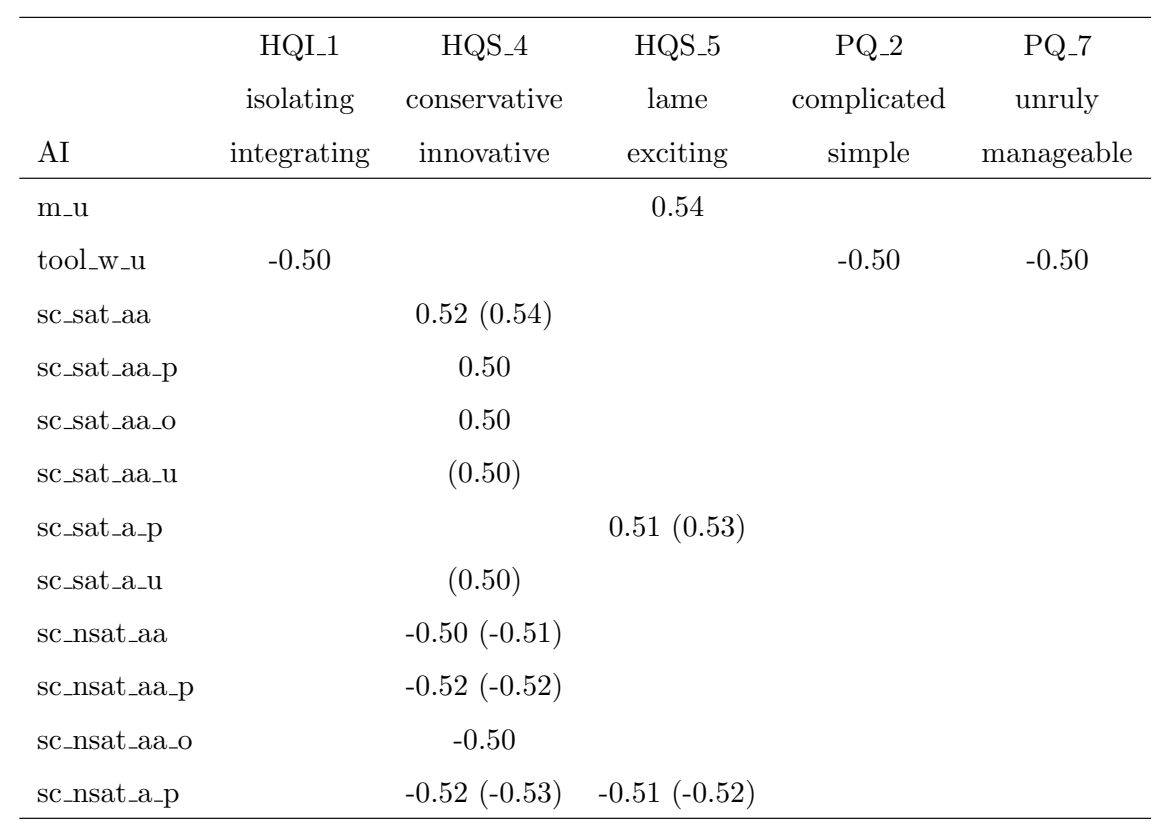

Figures 2, 3 and 4 show the correlation matrices corresponding to the coefficients obtained from Pearson, Spearman and Kendall tests respectively, providing a general overview of all the correlations in addition to those with large effect sizes in Table3. Matrices show that the more and stronger correlations are between typical-original (HQS_1), conservative-innovative (HQS_4) and lameexciting (HQS_5) attributes and those AIs generated through the expert evaluation of Web site compliance to WCAG 2.0, particularly again to those success criteria belonging to the Perceivable principle.

In order to check the consistency and robustness of the results we performed Pearson's correlation test using sampling with replacement. We applied the bootstrapping technique for different numbers of bootstrap replicates $(R=100$, $325500,1000,1500,5000,10000)$ for the same confidence level $(0.95)$. We obtained 


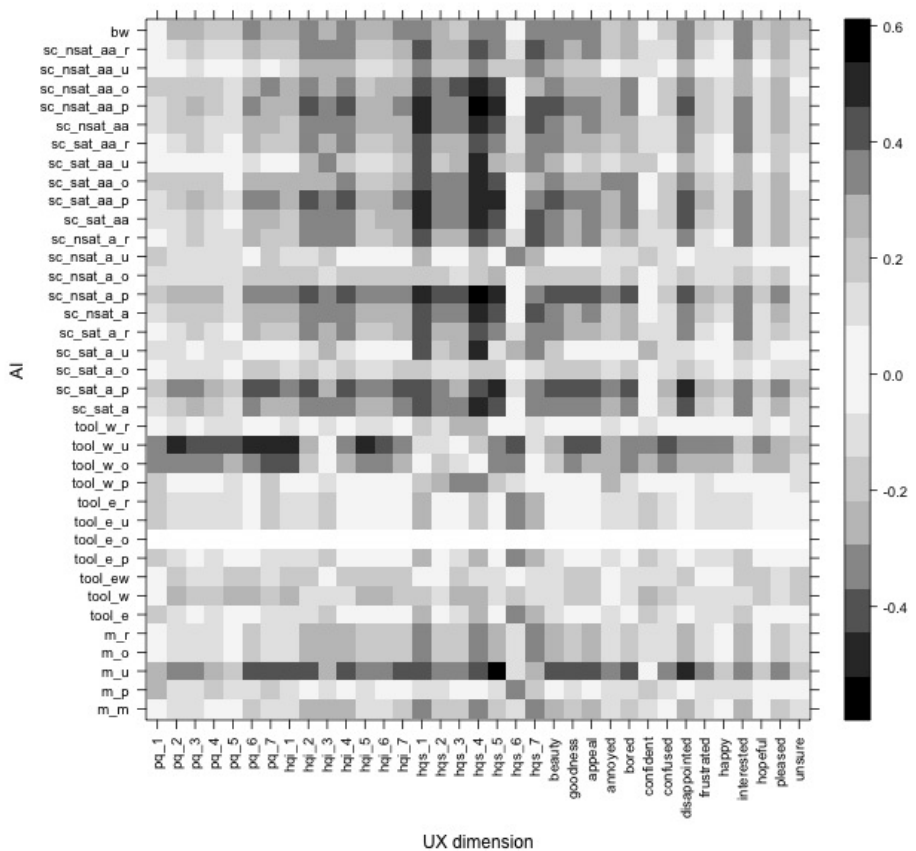

Figure 2: Correlation matrix for the AIs and UX attributes, using the Pearson test $[\mathrm{N}=88$, 11 participants $\mathrm{x} 4$ Web sites $\mathrm{x} 2$ Web pages, $p<0.001]$.

the bias (the difference between the mean of the $\mathrm{R}$ bootstrap samples and the original estimate), the standard error (the standard deviation of the $\mathrm{R}$ bootstrap samples) and the confidence intervals for $\mathrm{R}$ samples. We did not observe big differences on the confidence intervals depending on the size of R. On the other hand the bias and the standard error are very low, which suggests the similarity with the original estimate. For instance, if we take the bootstrap results for the correlation between the HQS_5 attribute and the sc_sat_aa AI, the obtained minimum and maximum values for the bias and the standard error were [-0.0094, 0.001], [0.066, 0.071] respectively. We also applied the bootstrapping technique by relaxing the confidence level $(0.90,0.92)$. In this case the confidence intervals kept stable even when increasing alpha. As the bias and the standard error obtained as a result of applying the bootstrapping technique is very low, none of the confidence intervals include the zero value and the range of the intervals 


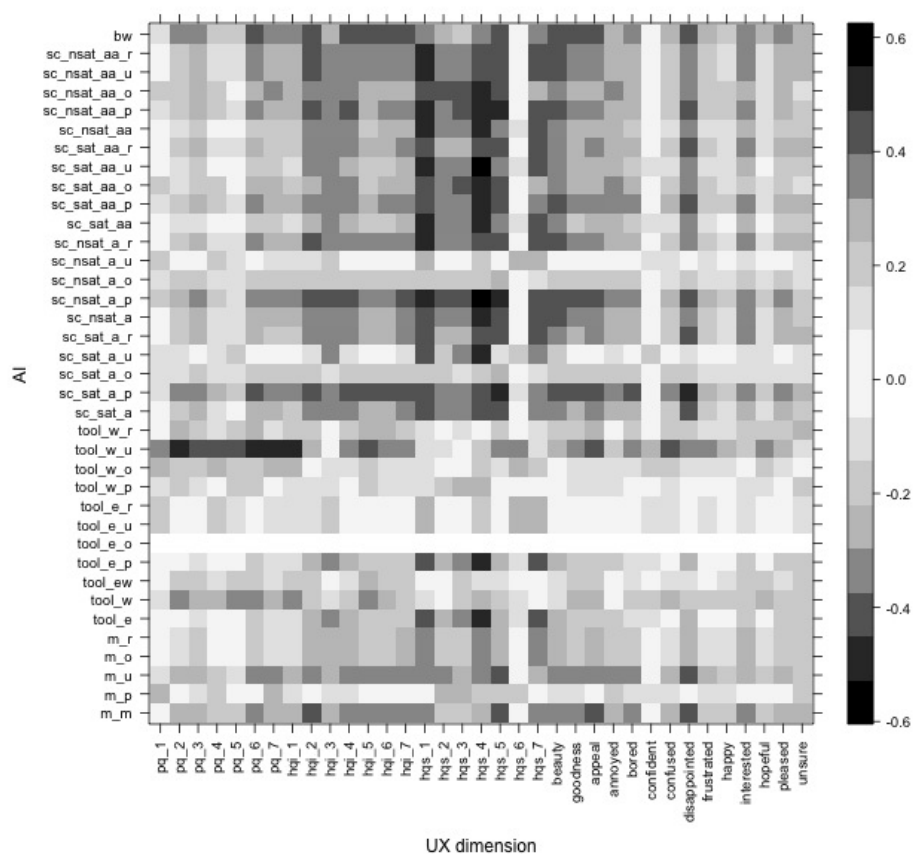

Figure 3: Correlation matrix for the AIs and UX attributes, using the Spearman test $[\mathrm{N}=88$, 11 participants $\mathrm{x} 4$ Web sites $\mathrm{x} 2$ Web pages, $p<0.001]$.

is not very wide we conclude that the correlations are robust.

\subsection{Analysis of the Interviews}

We looked into the transcriptions of the interviews in order to better understand the practical importance of the identified correlations and the reasons why they emerged. For this analysis we only focused on those three UX attributes typical-original (HQS_1), conservative-innovative (HQS_4) and lameexciting (HQS_5), which showed stronger and more consistent correlations across the three statistical tests we performed.

We queried the transcripts using the synonyms and antonyms of the wordpairs of the identified UX attributes. Then we annotated and coded the excerpts 


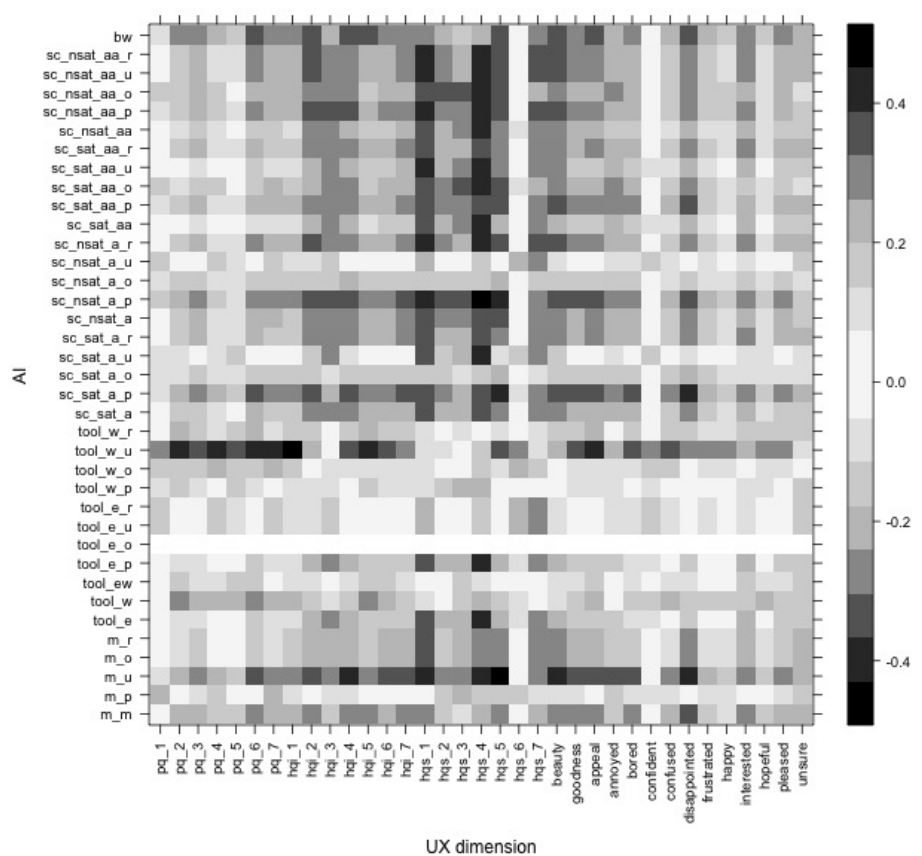

Figure 4: Correlation matrix for the AIs and UX attributes, using the Kendall test $[\mathrm{N}=88,11$ participants $\mathrm{x} 4$ Web sites $\mathrm{x} 2$ Web pages, $p<0.001$ ].

we retrieved. We used the Merriam-Webster online dictionary 7 , the Collins online dictionary ${ }^{8}$ and the qualitative data analysis software NVivo 10 for this analysis.

\subsubsection{Hedonic Quality-Stimulation: Typical-Original (HQS_1)}

Typical can be defined as being or serving as a representative example of a particular type, characteristic, having the qualities associated with the members of a particular group or kind. We looked up synonyms (such as archetypal, standard, model, normal, classic) and opposites (including unique, unusual, unexpected, exceptional) in the transcripts. The majority of comments were observations about situations that occur regularly such as coming across non-

\footnotetext{
${ }^{7}$ http://www.merriam-webster.com/thesaurus/

${ }^{8}$ http://www.collinsdictionary.com/dictionary/english-thesaurus
} 
accessible Web sites. Two expert users (P01, P02) said that finding accessible (...) (P05)"

Regarding unconventional layouts, the gastronomic information on W2 was conveyed through links in a hierarchical multi-level layout, instead of providing the information on the same page, which was not expected by P09: "The menu, 385 I was expecting a document, right? and then of course, it was not a menu as such, it was like a bunch of links". P02 said that it was unusual to find the link to the accessible version of the Web site quite at the beginning of the Web page on W1: "No, it's not usually the case (...) it's quite at the top". The fact that the content was placed before the navigation menu on W1 attracted P03 
participant's attention.

Apart from the aspects related to Web content and layout, some participants valued the novel experience of visiting a restaurant Web site for the first time: "First time I visit a restaurant Web site, I didn't know what it could contain and I really liked it because it was non-accessible but if you try, you get to know new things (P06-W1)". Many participants praised and linked originality and the accessibility of W1 and W2 Web sites although this was not always the case: "It's not original, it gets to the point and is very professional, it is not arty (...) the page lacks an artistic touch (P05-W2)". This suggests that not only the accessibility of a Web site, but the quality of the textual content provided on the Web site influences the perception of originality: "The text meets the objective of informing, and the more interesting the better, we ignore all the visual part of about the venue and the dishes so textual explanations are interesting for us (P10)".

\subsubsection{Hedonic Quality-Stimulation: Conservative-Innovative (HQS_4)}

This attribute is closely related to the previous one (typical-original, HQS_1) as many comments coded as original were also coded into the innovative category. Nevertheless, there is a subtle but important difference about the meaning: unlike HQS_1, HQS_4 deals more with conservatism and innovation, which are somehow related to progress and evolution.

410 Conservative represents a tendency to favour the preservation of established ideas, conditions, values or institutions, opposing innovation. Some synonyms are traditional, conventional, moderate, cautious and reactionary. While liberal, radical, progressive, innovative, and imaginative are some examples of opposites.

We found few examples of comments which relate both conservative and inaccessibility: "The feeling is that the page hasn't served me for anything (...) in addition to annoying me I feeling the site is totally conservative, it has no innovation, it is not accessible at all (P04-W3)". P05 goes further and suggests that the lack of accessibility is an indicator of non-evolved society or country: "I think they haven't thought much about people who are not able to see, right? 
Regarding accessibility, nowadays it should already be there (...) I'm sure that in the Netherlands these non-accessible Web sites aren't developed any more ...(P05-W3)".

Innovative relates to showing a noteworthy use of the imagination and creativity especially in creating new things and inventing. Synonyms include novel,

425 new, original, different, fresh, unusual and unfamiliar. Most comments who link innovation and accessibility refer to the W1 Web site: "Seeing a different design has aroused my curiosity (...) what I have liked most is its innovative character...(P10-W1)".

\subsubsection{Hedonic Quality-Stimulation: Lame-Exciting (HQS_5)}

Lame means falling short of a standard, painful or weak, unconvincing, not effective or enthusiastic, conventional or uninspiring. Synonyms include defective, unconvincing, poor, inadequate, weak, insufficient and unsatisfactory. Most comments were about the W3 Web site, where we found at least one comment for each participant about W3: "It is not easy to handle. The information is quite hard to find with a screen reader, unspecific (...) It's too much effort, you waste a lot of time, the information you get is not very reliable. The messy links were not very clarifying, they do not give an idea of the content in each link (...) it was like a labyrinth, too complicated (...) to get something specific you would have to invest much time, effort and I'm not sure if one would get to anything concrete (...) it forces you to have to do all the tour of the entire page. It's like to get a room in a hotel you would have to go through the 360 rooms it has (...) is the most difficult one of the four Web sites, for a screen reader user is unsatisfactory (POץ)".

We only found comments from one participant who would stress the lameness of W4: "Disappointed, confused in many moments, completely bored and very annoyed (P02)". We also found a few comments about the most accessible Web sites, W1 and W2: "It's not a very clear content as to the presented links. It's promising, it seems it will provide information, but the information that exposes is very literary. Very literary and very repetitive for a screen reader (P07-W1)". 

for exciting would be stimulating, inspiring, thrilling or sensational while opposites include boring, dull, dreary, monotonous, uninspiring. Most comments are about the accessible Web sites, W1 and W2: "I found the Web page attractive (...) a desirable place to go (...) a Web site with very specific and clear inforthe possibility to revisit the Web site and other even showed their willingness to go to the restaurant: "I'll check it at home, maybe I'll write them an email telling them that the Web site is perfect (P06-W2)".

\section{Discussion} cessible are related to perceptions such as creative, original, exciting and new. In contrast, Web sites considered to be non-accessible are related to perceptions like standard, typical, lame and commonplace. Findings also support that a 
positive accessibility perception is related to appraisals of goodness, appeal and pointment and frustration) than with technical terms about the Web, assistive technologies and accessibility. The identified attributes do not only serve as proxies for perceived Web accessibility, but they can also facilitate the commu- 
of blind users with a Web site.

\subsection{On the Relationship between UX Attributes and Web Accessibility Indica- tors}

We found evidence to support the relationship between the UX attributes corresponding to the hedonic quality-stimulation typical-original (HQS_1), conservativeinnovative (HQS_4), lame-exciting (HQS_5) and AIs representing the conformance to WCAG 2.0 guidelines. We observed a slight predominance of AIs corresponding to the Perceivable principle of WCAG 2.0. Accessible Web sites (in terms of a higher number of satisfied SC or fewer number of non-satisfied ones (in terms of a lower number of satisfied SC or higher number of non-satisfied SC) are perceived as typical, conservative and lame.

Comments of participants during the interviews provided additional evidence to support the correlations found between compliance to guidelines and three hedonic quality-stimulation attributes: original, innovative and exciting. Participants may consider accessible Web sites original because they still find many non-accessible Web sites on the Web. Expert users claimed there are still many non-accessible Web sites (especially restaurant Web sites) and coming across an accessible Web site is considered a novelty. In line with this, some users appreciated the uncommon event of encountering alternative and theoretically more accessible versions of Web sites. We also learned that the perception of originality is not only influenced by the Web site's accessibility: the quality of textual content and its arrangement boosted the perception of originality. Nevertheless, we are cautious about this statement as user expertise and familiarity with the domain seem to play the role of moderator variables.

Accessible Web sites were considered to be innovative and related to progress and evolution, while non-accessible ones were regarded as conservative. A clear relationship between lame and lack of accessibility as well as between exciting and accessibility was observed. With regard to lame, participants gave some 

serious accessibility problems: "a labyrinth, a loop going nowhere, trying each room of a hotel to select just one, a fortress for the accessibility, a bunker...". Conversely, participants were strongly motivated on accessible Web sites, which led to Web site revisitation or even to physically go to the restaurant featured the first time may have contributed to some extent to the motivation of participants. This suggests that the hedonic quality-stimulation is not only driven by the characteristics of the stimuli, but by other experiential aspects, such as expectations and previous experiences on the Web.

Nevertheless, we found it surprising the unbalanced number of comments about the lame attribute on the Web sites with low accessibility (W3 and W4). One possible explanation for this is that the severity of accessible barriers may have more impact than their number. For instance, W3 did not have proper text alternatives for the navigational image links, which had devastating concators were computed using the Barrier Walkthrough method and aesthetic judgements were made by sighted users. They found that Web pages judged on the classical aesthetics attribute as being visually clean showed significant correlations with accessibility. No correlation was found between the expressive 570 sequences on the hedonic quality-stimulation attributes. Even if the content about the gastronomic offer was accessible users were totally demotivated when exploring the homepage. On the other hand, the texts of the navigation menu link in W4 were clear and concise although the content about gastronomic offer was not completely accessible. This can be explained in light of previous works that highlighted the importance of the information architecture and the understandability of the texts in navigation menus. Blind users use them to get an overview of Web sites, which helps them form a mental model of the Web site (Leuthold et al. 2008).

Few works in the literature relate UX attributes and Web accessibility. One exception is the study by Mbipom \& Harper (2011) where accessibility indiaesthetic attributes and accessibility indicating that an expressive design is not 
necessarily in conflict with accessibility. In fact, expressive aesthetics Lavie \& Tractinsky, 2004 Hassenzahl, 2008) match with the hedonic quality-stimulation attribute from Hassenzahl's model. Specifically the original, innovative and exciting attributes map to original, creative and fascinating expressive aesthetics attributes respectively. Hence, our findings do not only corroborate that an expressive design is not necessarily in conflict with accessibility, but we provide additional evidence on the interplay between Web aesthetics and accessibility. In this context, we emphasise that web aesthetics should be conceived beyond the visual representation and content of Web sites. In order to increase the aesthetic perception of Web sites the information architecture and the quality of texts should be paid attention.

Whether compliance to accessibility guidelines implies a satisfying user experience is a controversial topic. Our findings suggest that compliance to guidelines benefits the original, innovative and exciting attributes of the hedonic quality-stimulation attribute. It seems reasonable to assume that an accessible Web site is more likely to offer users new impressions and opportunities than a non-accessible Web site. If the content of a Web site is accessible, users will have more chances to be stimulated and motivated to navigate on that Web site than on a poorly accessible Web site.

\subsection{Implications for Design}

The accessibility problems participants encountered are those covered by previous works and guidelines (Theofanos \& Redish, 2003, Leuthold et al., 2008 Leporini \& Paternò, 2008). These works provide already a substantial body of knowledge on the design recommendations to build more accessible Web sites. Our findings corroborate how critical information architecture and navigation menus are, how beneficial it is to provide 'skip navigation' links and the effect of text quality of the aesthetic perception of Web sites. As far as design recommendations are concerned, our findings stress the criticality of the mentioned features in that they do not only improve accessibility, but they also boost a positive perception of Web sites. 


\section{Conclusions}

In order to acquire a better understanding of the interplay between UX and Web accessibility, we analysed the relationships between UX attributes and perceived Web accessibility (PWA) and accessibility indicators derived from confor-

style. Future studies should address the possibility of generalising results to other type of stimuli. 


\section{Acknowledgements}

A copy of the Web sites, the results of their Web accessibility evaluations stimuli. A. Aizpurua held a Ph.D. scholarship from The Department of Education, Universities and Research of the Basque Government when the study was conducted. The work has been partly funded by the same department under the Grant IT395-10 and the Basque Advanced Informatics Laboratory (BAILab) at

\section{References}

Abascal, J., Arrue, M., Fajardo, I., Garay, N., \& Tomás, J. (2004). The use of guidelines to automatically verify web accessibility. Universal Access

Aizpurua, A., Arrue, M., \& Vigo, M. (2013). Uncovering the role of expectations on perceived web accessibility. In Proceedings of the 15th International ACM SIGACCESS Conference on Computers and Accessibility ASSETS '13 (pp. 1145/2513383.2513411. doi $10.1145 / 2513383.2513411$.

Aizpurua, A., Arrue, M., \& Vigo, M. (2015). Prejudices, memories, expectations and confidence influence experienced accessibility on the web. Computers in

п Human Behavior, 51, Part A, 152 -160. URL: http://www.sciencedirect.

n com/science/article/pii/S0747563215003222 doi/http://dx.doi.org/ $10.1016 / \mathrm{j}$.chb.2015.04.035

Bargas-Avila, J. A., \& Hornbæk, K. (2011). Old wine in new bottles or novel challenges: A critical analysis of empirical studies of user experience. In Proceedings of the SIGCHI Conference on Human Factors in Computing Systems n CHI '11 (pp. 2689-2698). New York, NY, USA: ACM. URL: http://doi. 655 
Bigham, J. P., Cavender, A. C., Brudvik, J. T., Wobbrock, J. O., \& Lander, R. E. (2007). Webinsitu: A comparative analysis of blind and sighted browsing behavior. In Proceedings of the 9th International ACM SIGACCESS Conference on Computers and Accessibility Assets '07 (pp. 51-58). New York, NY, USA: ACM. URL: http://doi.acm.org/10.1145/1296843.1296854 doi:10.1145/1296843.1296854

Brajnik, G. (2006). Web accessibility testing: When the method is the culprit. In ICCHP, LNCS 4061 (pp. 156-163). Springer Verlag.

Caldwell, B., Cooper, M., Reid, L. G., \& Vanderheiden, G. (2008). Web con665

tent accessibility guidelines (WCAG) 2.0. URL: http://www.w3.org/TR/ WCAG20/.

Carifio, J., \& Perla, R. J. (2007). Ten Common Misunderstandings, Misconceptions, Persistent Myths and Urban Legends about Likert Scales and Likert Response Formats and their Antidotes. Journal of Social Sciences, 3, 106116.

Chisholm, W., Vanderheiden, G., \& Jacobs, I. (1999). Web content accessibility guidelines (WCAG) 1.0. URL: http://www.w3.org/TR/WAI-WEBCONTENT/.

Clark-Carter, D. (2004). Quantitative Psychological Research: A Student's Handbook. Psychology Press.

De Marsico, M., \& Levialdi, S. (2004). Evaluating web sites: Exploiting user's expectations. The International Journal of Human-Computer Studies, 60, 381-416. URL: http://dx.doi.org/10.1016/j.ijhcs.2003.10.008 doi:10.1016/j.ijhcs.2003.10.008.

Gay, G., \& Li, C. Q. (2010). Achecker: Open, interactive, customizable, 680 web accessibility checking. In Proceedings of the 2004 International Crossdisciplinary Workshop on Web Accessibility (W4A) W4A (pp. 23:1-23:2).

口 New York, NY, USA: ACM. URL: http://doi.acm.org/10.1145/1805986. 1806019, doi $10.1145 / 1805986.1806019$. 
van der Geest, T., van der Meij, H., \& van Puffelen, C. (2014). Self-assessed and actual internet skills of people with visual impairments. Universal Access in

the Information Society, 13, 161-174. URL: http://dx.doi.org/10.1007/ s10209-013-0304-5. doi $10.1007 /$ s10209-013-0304-5.

Hassenzahl, M. (2005). The thing and i: Understanding the relationship between user and product. In Funology (pp. 31-42). Springer Netherlands volume 3 of

690 „Human-Computer Interaction Series. URL: http://dx.doi.org/10.1007/ 1-4020-2967-5_4

Hassenzahl, M. (2008). The interplay of beauty, goodness, and usability in interactive products. Human-Computer Interaction, 19, a 319-349. URL: http://dx.doi.org/10.1207/s15327051hci1904_2. doi.10. 1207/s15327051hci1904_2.

ISO (1998). Iso 9241-11: Ergonomic requirements for office work with visual display terminals (vdts). part 11: Guidance on usability.

Lavie, T., \& Tractinsky, N. (2004). Assessing dimensions of perceived visual aesthetics of web sites. The International Journal of Human-Computer Studies, 60, 269-298. URL: http://dx.doi.org/10.1016/j.ijhcs.2003.09.002 doi:10.1016/j.ijhcs.2003.09.002.

Law, E. L.-C., Roto, V., Hassenzahl, M., Vermeeren, A. P., \& Kort, J. (2009). Understanding, scoping and defining user experience: A survey approach. In Proceedings of the SIGCHI Conference on Human Factors in Computing 705 \Systems (pp. 719-728). New York, NY, USA: ACM. URL: http://doi.acm. org/10.1145/1518701.1518813 doi $10.1145 / 1518701.1518813$.

Lazar, J., Feng, J., \& Allen, A. (2006). Determining the impact of computer frustration on the mood of blind users browsing the web. In Proceedings of the 8th International ACM SIGACCESS Conference on Computers and Accessi710 bility Assets '06 (pp. 149-156). New York, NY, USA: ACM. URL: http:// doi.acm.org/10.1145/1168987.1169013 doi 10.1145/1168987.1169013. 
Leporini, B., \& Paternò, F. (2008). Applying web usability criteria for vision-impaired users: Does it really improve task performance? International Journal of Human-Computer Interaction, 24, 17-47. URL: http://dx. doi.org/10.1080/10447310701771472 doi:10.1080/10447310701771472 arXiv:http://dx.doi.org/10.1080/10447310701771472

Leuthold, S., Bargas-Avila, J. A., \& Opwis, K. (2008). Beyond web content accessibility guidelines: Design of enhanced text user interfaces for blind internet users. International Journal of Human-Computer Studies, 66, 257 - 270. URL: http://www.sciencedirect.com/science/article/pii/

(1071581907001413 doi http://dx.doi.org/10.1016/j.ijhcs.2007.10. 006 .

Lindley, S. E., Meek, S., Sellen, A., \& Harper, R. (2012). "it's simply integral to what i do": Enquiries into how the web is weaved into everyday life. In Proceedings of the 21st International Conference on World Wide Web WWW q '12 (pp. 1067-1076). New York, NY, USA: ACM. URL: http://doi.acm. org/10.1145/2187836.2187979 doi $10.1145 / 2187836.2187979$.

Lopes, R., Gomes, D., \& Carriço, L. (2010). Web not for all: A large scale study of web accessibility. In Proceedings of the 2010 International Cross Disciplinary Conference on Web Accessibility (W4A) W4A '10 (pp. 10:110:4). New York, NY, USA: ACM. URL: http://doi.acm.org/10.1145/ 1805986.1806001 doi $10.1145 / 1805986.1806001$.

Maxwell, K. (2006). What you need to know about statistics. In Web Engineer1 ing (pp. 365-408). Springer Berlin Heidelberg. URL: http://dx.doi.org/ $735 \quad 10.1007 / 3-540-28218-1 \_12$ doi $10.1007 / 3-540-28218-1 \_12$.

Mbipom, G., \& Harper, S. (2011). The interplay between web aesthetics and accessibility. In The Proceedings of the 13th International ACM SIGACCESS Conference on Computers and Accessibility ASSETS '11 (pp. 147-154).

口 New York, NY, USA: ACM. URL: http://doi.acm.org/10.1145/2049536. 2049564. doi $10.1145 / 2049536.2049564$. 
Murphy, E., Kuber, R., McAllister, G., Strain, P., \& Yu, W. (2008). An empirical investigation into the difficulties experienced by visually impaired internet users. Universal Access in the Information Society, 7, 79-

91. URL: http://dx.doi.org/10.1007/s10209-007-0098-4 doi 10.1007/ s10209-007-0098-4

Norman, G. (2010). Likert scales, levels of measurement and the laws of statisq tics. Advances in Health Sciences Education, 15, 625-632. URL: http://dx. doi.org/10.1007/s10459-010-9222-y, doi 10.1007/s10459-010-9222-y.

Petrie, H., Hamilton, F., \& King, N. (2004). Tension, what tension?: Website accessibility and visual design. In Proceedings of the 2004 International Crossdisciplinary Workshop on Web Accessibility (W4A) W4A '04 (pp. 13-18).

口 New York, NY, USA: ACM. URL: http://doi.acm.org/10.1145/990657. 990660, doi $10.1145 / 990657.990660$.

Petrie, H., \& Kheir, O. (2007). The relationship between accessibility and usability of websites. In Proceedings of the SIGCHI Conference on Human Factors in Computing Systems CHI '07 (pp. 397-406). New York, NY, USA: ACM. URL: http://doi.acm.org/10.1145/1240624.1240688 doi:10.1145/1240624.1240688

Petrie, H., \& Precious, J. (2010). Measuring user experience of websites: Think aloud protocols and an emotion word prompt list. In CHI '10 Extended Abstracts on Human Factors in Computing Systems CHI EA '10 (pp. 3673q 3678). New York, NY, USA: ACM. URL: http://doi.acm.org/10.1145/ 1753846.1754037, doi $10.1145 / 1753846.1754037$.

Power, C., Freire, A., Petrie, H., \& Swallow, D. (2012). Guidelines are only half of the story: Accessibility problems encountered by blind users on the web. In Proceedings of the SIGCHI Conference on Human Factors in Computing 口 Systems CHI '12 (pp. 433-442). New York, NY, USA: ACM. URL: http:// doi.acm.org/10.1145/2207676.2207736. doi 10.1145/2207676.2207736. 
Theofanos, M. F., \& Redish, J. G. (2003). Bridging the gap: Between acces770 n sibility and usability. interactions, 10, 36-51. URL: http://doi.acm.org/ 10.1145/947226.947227, doi:10.1145/947226.947227

Thüring, M., \& Mahlke, S. (2007). Usability, aesthetics and emotions in humantechnology interaction. International Jour-

口 nal of Psychology, 42, 253-264. URL: http://dx.doi.org/ $10.1080 / 00207590701396674$ doi $10.1080 / 00207590701396674$. arXiv:http://dx.doi.org/10.1080/00207590701396674.

Vigo, M., Arrue, M., Brajnik, G., Lomuscio, R., \& Abascal, J. (2007). Quantitative metrics for measuring web accessibility. In Proceedings of the 2004 International Cross-disciplinary Workshop on Web Accessibility (W4A) W4A

'07 (pp. 99-107). New York, NY, USA: ACM. URL: http://doi.acm.org/ $10.1145 / 1243441.1243465$, doi $10.1145 / 1243441.1243465$.

Vigo, M., \& Harper, S. (2013). Coping tactics employed by visually disabled users on the web. The International Journal of Human-Computer Studies, 71 , 1013-1025. URL: http://dx.doi.org/10.1016/j.ijhcs.2013.08.002 doi:10.1016/j.ijhcs.2013.08.002.

Yesilada, Y., Brajnik, G., Vigo, M., \& Harper, S. (2012). Understanding web accessibility and its drivers. In Proceedings of the International CrossDisciplinary Conference on Web Accessibility W4A '12 (pp. 19:1-19:9). New

\ York, NY, USA: ACM. URL: http://doi.acm.org/10.1145/2207016. 